\title{
Comparison of gray matter volume between migraine and "strict-criteria" tension-type headache
}

\author{
Wei-Ta Chen ${ }^{1,2,3,4^{*}}$ (D), Kun-Hsien Chou ${ }^{3,5}$, Pei-Lin Lee ${ }^{6}$, Fu-Jung Hsiao ${ }^{3}$, David M. Niddam³,4, Kuan-Lin Lai ${ }^{1,2}$, \\ Jong-Ling Fuh ${ }^{1,2,3}$, Ching-Po Lin ${ }^{3,4,5,6}$ and Shuu-Jiun Wang ${ }^{1,2,3,4}$
}

\begin{abstract}
Background: Despite evidently distinct symptoms, tension-type headache (TTH) and migraine are highly comorbid and exhibit many similarities in clinical practice. The purpose of this study was to investigate whether both types of headaches are similar in brain morphology.

Methods: Consecutive patients with $\mathrm{TH}$ and age- and sex-matched patients with migraine and healthy controls were enrolled for brain magnetic resonance imaging examination. Patients with TTH were excluded if they reported any headache features or associated symptoms of migraine. Changes in gray matter (GM) volume associated with headache diagnosis (TH vs. migraine) and frequency (episodic vs. chronic) were examined using voxel-based morphometry. The correlation with headache profile and the discriminative ability between TTH and migraine were also investigated for these GM changes.

Results: In comparison with controls $(n=43)$, the patients with $T \mathrm{TH}$ (25 episodic and 24 chronic) exhibited a GM volume increase in the anterior cingulate cortex, supramarginal gyrus, temporal pole, lateral occipital cortex, and caudate. The patients with migraine (31 episodic and 25 chronic) conversely exhibited a GM volume decrease in the orbitofrontal cortex. These GM changes did not correlate with any headache profile. A voxel-wise $2 \times 2$ factorial analysis further revealed the substantial effects of headache types and frequency in the comparison of GM volume between $T \mathrm{TH}$ and migraine. Specifically, the migraine group (vs. TTH) had a GM decrease in the superior and middle frontal gyri, cerebellum, dorsal striatum, and precuneus. The chronic group (vs. episodic group) otherwise demonstrated a GM decrease in the bilateral insula and anterior cingulate cortex. In receiver operating characteristic analysis, the GM volumes of the left superior frontal gyrus and right cerebellum $V$ combined had good discriminative ability for distinguishing TTH and migraine (area under the curve $=0.806$ ).

Conclusions: TTH and migraine are separate headache disorders with different characteristics in relation to GM changes. The major morphological difference between the two types of headaches is the relative GM decrease of the prefrontal and cerebellar regions in migraine, which may reflect a higher allostatic load associated with this disabling headache.
\end{abstract}

Keywords: Tension-type headache, Migraine, Gray matter, Voxel-based morphometry, Magnetic resonance imaging (MRI)

\footnotetext{
* Correspondence: wtchen@vghtpe.gov.tw

'Department of Neurology, School of Medicine, National Yang-Ming

University, Taipei, Taiwan

${ }^{2}$ The Neurological Institute, Taipei Veterans General Hospital, Taipei, Taiwan

Full list of author information is available at the end of the article
}

照 Springer Open

(c) The Author(s). 2018 Open Access This article is distributed under the terms of the Creative Commons Attribution 4.0 International License (http://creativecommons.org/licenses/by/4.0/), which permits unrestricted use, distribution, and reproduction in any medium, provided you give appropriate credit to the original author(s) and the source, provide a link to the Creative Commons license, and indicate if changes were made. 


\section{Background}

Tension-type headache (TTH) and migraine are both common headache disorders. In a Danish population survey, the prevalence of migraine did not change significantly (11-15\%) during a 12-year period, whereas the prevalence of TTH (79-87\%) increased significantly and was higher than that of migraine [1]. In the Global Burden of Diseases, Injuries, and Risk Factors Study 2016, tension type headache ranks third in terms of global prevalence, and sixth in terms of global incidence, both being higher than that of migraine [2]. Despite the high prevalence and incidence of TTH, scientific interest in TTH has long been sparse probably because the pain intensity, associated symptoms and functional impairment caused by TTH are relatively mild compared with those caused by migraine.

According to the International Classification of Headache Disorders, third edition beta (ICHD-III beta) [3], the characteristics of TTH and migraine are undeniably distinct. Migraine headache is typically unilateral, pulsatile, moderate-to-severe in intensity and aggravated by daily activities. By contrast, TTH is bilateral, nonpulsatile, and mild-to-moderate in intensity and not aggravated by daily activities. In clinical practice, however, TTH and migraine have many similarities, including common triggers, psychiatric comorbidities and responsiveness to similar medications [4]. Moreover, migraine and TTH usually coexist in the same patient [1], which may result in individuals with TTH being clinically diagnosed and treated as more disabling migraine. In the Spectrum Study, 37\% of patients initially diagnosed with TTH were later revealed to have migraine or migrainous headache [5]. A recent study using taxometric analysis did not support that TTH and migraine are separate clinical entities [6]. In pathophysiology, both types of headaches have been characterized by central sensitization, as revealed in many neurophysiological studies [7, 8]. A brain magnetic resonance imaging (MRI) study on patients with chronic TTH ( $\geq 15$ headache days/month) demonstrated a gray matter (GM) decrease in the anterior cingulate, insula, orbitofrontal cortex, dorsal pons, and other structures of the pain processing network [9]. These structural changes have also been documented in patients with migraine [10-13]. TTH and migraine thus seem more inter-related than would be suggested by their diagnostic criteria.

However, the findings suggesting a common pathophysiology for TTH and migraine must be confirmed without interference from migraine comorbidity. Notably, a study reported normal interictal plasma levels of calcitonin gene-related peptide (CGRP) in patients with chronic TTH; however, in the patient subgroup with pulsating pain quality, the CGRP level was higher, as in the group of patients with interictal migraine [14]. These findings implied that the presence of even one migrainous feature in patients with TTH may eventually lead to an association with migraine in the pathophysiology. In addition, some studies on quantitative sensory testing [15], brainstem excitability [16], laser evoked potentials [17], and temporal discrimination thresholds [18] have congruently revealed different somatosensory information processing between TTH and migraine, although migraine comorbidity was not deliberately excluded in patients with TTH. The present study thus hypothesized that TTH and migraine are different in brain morphology, which may reflect their distinct symptomatology, sensory processing, and disease burden. To test this hypothesis, we used strict criteria to enroll patients with "pure" TTH instead of relatively loose criteria, as suggested by ICHD-III beta (see Methods for details). Brain morphology was analyzed using voxel-based morphometry (VBM), a technique that has been widely employed to evaluate brain morphological alternations in various chronic pain syndromes including migraine [19]. To our knowledge, two MRI studies $[9,20]$ have employed VBM to investigate the brain morphological change in TTH. A study on episodic TTH $(<15$ headache days/ month) [20] did not reveal any GM change, whereas a study on chronic TTH, as aforementioned, suggested reduced GM in the pain processing network [9]. Few studies have compared the brain morphological differences between TTH and migraine. Clarifying these issues may facilitate the development of a TTH-specific therapy or brain signature.

\section{Methods}

\section{Participants}

Consecutive patients with episodic or chronic TTH were recruited from the Headache Clinic of Taipei Veterans General Hospital. For data comparison, this study also enrolled patients with episodic migraine (without aura) and chronic migraine, and healthy controls. The diagnosis of episodic migraine (code 1.1) and chronic migraine (code 1.3) was made according to the ICHD-III beta criteria [3]. Episodic and chronic TTH were also diagnosed based on the ICHD-III beta criteria, but a strict version was used instead-all patients were required to fulfill all (rather than $\geq 2$ ) of the following four headache characteristics, which are defined as the core syndromes of TTH: bilateral, mild-to-moderate intensity, non-pulsating and not aggravated by routine physical activity. Moreover, patients were required to report no migrainous features (nausea, vomiting, photophobia, or phonophobia) associated with their headaches, although the original ICHD-III beta criteria allow for the presence of either photophobia or phonophobia. Patients with episodic TTH reported 1-14 headache days/month (i.e., frequent episodic TTH, code 2.2), whereas patients with chronic TTH (code 2.3) 
experienced $\geq 15$ headache days/month for at least 3 months. Patients fulfilling the criteria of medication overuse headache (code 8.2) were excluded. Healthy controls did not have past or family histories of headache or any headache attacks in the previous year. All participants were right-handed, denied any history of systemic or neurologic disease, and presented with normal physical and neurological examinations. Participants who used any medications (including headache prophylactic drugs) or hormone therapy daily before participation were excluded. The hospital's Institutional Review Board approved the study protocol and each participant provided written informed consent.

At the first visit, all patients completed a semi-structured questionnaire on their demographics and headache profiles. They also completed a headache diary for at least 3 months after recruitment. The Migraine Disability Assessment (MIDAS) questionnaire assessed headache-related disability [21]. Depression was evaluated using the Beck Depression Inventory (BDI) [22].

Each participant underwent a scheduled MRI examination during the interictal period, which was defined as the absence of any headache within the 2 days before (days -1 and -2 ) and after (days +1 and +2 ) the MRI examination (day 0). The MRI examination was rescheduled when an acute headache attack occurred during this period, or when analgesics, triptans, or ergots were used for any reason within the $48 \mathrm{~h}$ preceding the examination. Notably, the presence of background or interval headache during the defined interictal period was permitted in patients with chronic migraine. For patients with chronic TTH, we did not synchronize all the MRI examinations in the interictal periods because some patients experienced almost daily or continuous headaches, and interictal imaging was thus impracticable. The temporal relationship between MRI examination and headache attacks was determined through the headache diary and follow-up phone calls.

\section{MRI protocol}

All participants underwent a three-dimensional wholebrain T1-weighted anatomical scan on a $3.0 \mathrm{~T}$ wholebody MRI scanner (Siemens Magnetom Tim Trio, Erlangen, Germany) equipped with a 32-channel phase array head coil at National Yang-Ming University, Taipei, Taiwan. For the acquisition of T1-weighted anatomical images, a sagittal multi-planar rapid acquire gradient echo sequence was employed with the following scanning parameters: repetition time $=2530 \mathrm{~ms}$, echo time $=3.0 \mathrm{~ms}$, inversion time $=1100 \mathrm{~ms}$, flip angle $=7^{\circ}, 192$ sagittal slices (without inter-slice gap and interpolation), number of excitations $=1$, field-of-view $=224 \times 256 \mathrm{~mm}^{2}$, matrix size $=224 \times 256$, and isotropic $1.0 \mathrm{~mm}^{3}$ resolution. All images were acquired parallel to the plane connecting the anterior and posterior commissure, and were visually assessed for image artifacts and significant motion problems.

\section{VBM analysis of brain GM}

Voxel-wised GM volume estimates were calculated using the VBM analysis framework [23] with the VBM8 toolbox (version 445, http://dbm.neuro.uni-jena.de/vbm.html), Statistical Parametric Mapping software (SPM8 version 6313, Wellcome Institute of Neurology, University College London, UK, http://www.fil.ion.ucl.ac.uk/spm/) and Matlab R2010a (Mathworks, Natick, MA, USA). The image preprocessing pipeline has been detailed in other studies [24, 25]. Before tissue segmentation, the image origin was set automatically using the center of mass approach with the VBM8 toolbox. This step reduced between-subject variability that may have confounded the subsequent tissue segmentation and image registration. Then, the native-space T1 anatomical images were corrected for field inhomogeneity and further partitioned into GM, white matter and cerebrospinal fluid compartments. To account for differences in global brain volume across the study participants, individual native-space tissue segments were further affine-aligned into the Montreal Neurological Institute (MNI) space. For inter-subject registration, these affine-aligned GM and white matter images were warped to a study-specific tissue template constructed from all study participants using a diffeomorphic non-linear image registration algorithm [26]. Subsequently, the normalized GM tissue images were modulated by the Jacobian determinants of the deformation field to preserve the local tissue volume estimation during the non-linear image deformation process. These modulated GM tissue images were spatially smoothed using an isotropic Gaussian kernel with 8-mm full-width at half-maximum and served as the inputs for the voxelwise statistical analyses. To create the explicit mask for the voxel-wise statistical analyses, unmodulated GM images in the MNI space were also obtained and averaged across all the study participants. In construction of the final consensus mask, the voxels with GM tissue probabilities lower than a threshold value of 0.2 were excluded to minimize potential edge effects between different tissue types.

\section{Statistical analysis of neuroimaging data}

Voxel-wise statistical analyses and region of interest (ROI) analyses were conducted using the generalized linear model Flex toolbox (http://mrtools.mgh.harvard.edu/ index.php?title=GLM_Flex) and SPSS software (version 17, SPSS, Chicago, IL, USA) respectively. For all voxelwise statistical analyses, the results revealed significant effects at the cluster-level family-wise-error corrected $P$ value of $<0.05$, with a cluster forming threshold of a 
voxel-level $P$ value of $<0.005$, and 271 voxel extents. This statistical criterion was determined based on the empirical results of a Monte Carlo simulation using 3dClustSim (permutations $=10,000$; with explicit GM mask; version AFNI_17.1.04). In addition to the thresholded results reported as the major findings in this manuscript, we have also uploaded all the un-thresholded statistical maps to the NeuroVault website, available through the following permanent link: https://neurovault.org/collections/3198/. In this study, preprocessed whole-brain GM tissue segments and the mean GM volumes of specific ROIs were used to address the following three research questions:

\section{Question 1: Is the GM volume differed in patients with TTH} and migraine (vs. controls) and linked to headache profiles? To determine the GM volume difference between controls and patients with TTH, or migraine, a statistical design of voxel-wise single-factor three-level (TTH, migraine, and controls) analysis of covariance (ANCOVA) was employed, with age, sex, and BDI entered as nuisance variables. There were four contrasts for this statistical design: controls > TTH; controls < TTH; controls > migraine; controls < migraine. To investigate the clinical relevance, the GM volumes of the clusters with a significant between-group main effect (TTH vs. controls or migraine vs. controls) were further extracted, averaged, and correlated with headache profile (TTH or migraine, dependent on the contrast) using Spearman's rank order correlation. Participants' age, sex, and BDI were also included as confounding covariates in the correlation analyses.

\section{Question 2: Is the GM volume different between TTH and migraine and modulated by headache frequency?}

A voxel-wise $2 \times 2$ factorial design with the factors TYPE (TTH vs. migraine) and FREQUENCY (episodic vs. chronic headache) was used to examine the main effects of TYPE and FREQUENCY and their interaction. Age, sex and BDI were also entered as covariates of no interest. In the post-hoc analysis, the GM volume was compared between TTH and migraine in their episodic forms (episodic TTH vs. episodic migraine) and chronic forms (chronic TTH vs. chronic migraine), respectively.

\section{Question 3: Is GM difference predictive of the headache type?}

The GM volumes indicating group differences between headache diagnoses (TTH vs. migraine) were further analyzed using a logistic regression model which adjusted for age, sex, and BDI to confirm the significance of the headache type prediction (TTH vs. migraine). The predictive value of the regression model was further estimated using the area under the receiver operating characteristic (ROC) curve.
The threshold for statistical significance was a $P$ value of $<0.05$ (two-tailed) throughout the study.

\section{Results}

Demographics and clinical profiles

A total of 156 individuals participated in this study: 43 controls, 56 patients with migraine (31 with episodic and 25 with chronic migraine), and 57 patients with TTH (30 with episodic and 27 with chronic TTH). Of the 57 patients with TTH, eight (five with episodic and three with chronic TTH) were excluded because of the presence of migrainous features according to their headache diary. The remaining 49 patients (25 with episodic and 24 with chronic TTH) became our final TTH group. The three groups did not differ in age and sex; however, BDI score was higher in the migraine $(P<0.001$ vs. controls $)$ and TTH $(P=0.020$ vs. controls) groups (Table 1$)$. Patients with migraine and TTH did not differ in average disease duration or headache frequency, but headache intensity, MIDAS score, and analgesics use frequency-as expected-were all higher in the migraine group than in the TTH group (all $P<0.05$ ). The demographics and clinical parameters of the five participant groups (controls vs. episodic migraine vs. chronic migraine vs. episodic TTH vs. chronic TTH) are shown in Additional file 1: Table S1.

Table 1 Demographics and clinical profile of the three participant groups

\begin{tabular}{|c|c|c|c|}
\hline & \multicolumn{3}{|l|}{ Group } \\
\hline & $\begin{array}{l}\text { Control } \\
(n=43)\end{array}$ & $\begin{array}{l}\text { Migraine } \\
(n=56)\end{array}$ & $\begin{array}{l}\text { TTH } \\
(n=49)\end{array}$ \\
\hline Age & $36.2 \pm 7.7$ & $37.5 \pm 7.6$ & $39.0 \pm 12.0$ \\
\hline Gender & $28 \mathrm{~F} / 15 \mathrm{M}$ & $37 F / 19 M$ & $26 \mathrm{~F} / 23 \mathrm{M}$ \\
\hline Episodic/chronic & - & $31 / 25$ & $25 / 24$ \\
\hline Headache frequency (d/mo) & - & $13.8 \pm 10.5$ & $14.0 \pm 10.6$ \\
\hline Disease duration (mo) & - & $194.6 \pm 116.7$ & $156.1 \pm 144.5$ \\
\hline Headache intensity $(0-10)^{a}$ & - & $5.9 \pm 2.1$ & $3.5 \pm 1.3$ \\
\hline $\operatorname{MIDAS}(0-270)^{a}$ & - & $26.1 \pm 35.8$ & $8.9 \pm 16.8$ \\
\hline BDI (0-63) b,c & $4.2 \pm 4.8$ & $8.7 \pm 5.7$ & $7.3 \pm 5.0$ \\
\hline \multicolumn{4}{|l|}{ Analgesics use profile } \\
\hline Frequency $(\mathrm{d} / \mathrm{mo})^{\mathrm{a}}$ & - & $4.4 \pm 2.5$ & $1.6 \pm 2.5$ \\
\hline \multicolumn{4}{|c|}{ Types of analgesics (\% of patients) } \\
\hline Simple analgesics & & $16.1 \%$ & $12.2 \%$ \\
\hline Compound analgesics & & $5.4 \%$ & $4.0 \%$ \\
\hline NSAIDs & & $8.9 \%$ & $6.1 \%$ \\
\hline Ergots & & $5.4 \%$ & $0 \%$ \\
\hline Triptans & & $5.4 \%$ & $0 \%$ \\
\hline
\end{tabular}

$B D I$ Beck Depression Inventory, $d$ days, MIDAS migraine disability assessment, mo month, NSAIDs Nonsteroidal anti-inflammatory drugs, $\Pi \mathrm{TH}$ tension-type headache

${ }^{a} p<0.05$ for migraine vs. TTH

${ }^{\mathrm{b}} p<0.05$ for migraine vs. control

$c^{c} p<0.05$ for TTH vs. control 


\section{Altered GM volume in patients with $\mathrm{TTH}$ and migraine (vs. controls)}

GM volume was increased in patients with TTH whereas it was decreased in patients with migraine compared with the controls (Table 2 and Fig. 1). In patients with TTH, GM volume was increased in the right caudate, temporal pole, left anterior cingulate cortex, supramarginal gyrus, and lateral occipital cortex. In the migraine group, GM volume was decreased only in the right orbitofrontal cortex. In the subgroup analysis, GM volume (vs. controls) was unaltered in the episodic and chronic migraine groups, but was increased in specific brain regions in patients with episodic TTH (the right caudateputamen, temporal pole, cerebellum, left anterior cingulate cortex, superior and middle frontal gyrus, and lateral occipital cortex) and chronic TTH (the right caudate, left supramarginal gyrus, and lateral occipital cortex) (Additional file 2: Figure S1). None of the aforementioned GM changes were correlated with headache profile.

\section{Altered GM volume in different headache types (TTH vs. migraine) and frequencies (episodic vs. chronic)}

A voxel-wise $2 \times 2$ factorial analysis revealed the significant effects of headache type, frequency, and their interaction in the comparison of GM volume between patients with TTH and migraine (Table 3 and Fig. 2). In the effect of headache type (TTH vs. migraine), GM volume was lower for the migraine group in the bilateral putamen, cerebellum, right caudate, putamen, precuneus, middle frontal gyrus, and left superior frontal gyrus. Regarding the effect of headache frequency (episodic vs. chronic), GM volume was lower for the chronic group (TTH and migraine combined) in the bilateral insula, right anterior cingulate cortex, and cerebellum. A significant headache type $\times$ frequency interaction was discovered in the right lateral occipital cortex.

Table 2 Altered gray matter volume in patients with migraine and $T \mathrm{TH}$

\begin{tabular}{|c|c|c|c|c|c|}
\hline \multicolumn{3}{|c|}{ MNI coordinates } & \multirow{2}{*}{$\begin{array}{l}\text { Cluster } \\
\text { size }\end{array}$} & \multirow[t]{2}{*}{ Anatomical region } & \multirow[t]{2}{*}{ Local peak T-value } \\
\hline$x$ & y & z & & & \\
\hline \multicolumn{6}{|c|}{ Migraine $<$ controls } \\
\hline 6 & 23 & -24 & 287 & R orbitofrontal cortex & 4.05 \\
\hline \multicolumn{6}{|c|}{$\mathrm{TTH}>$ controls } \\
\hline-11 & -80 & 51 & 1316 & L lateral occipital cortex & -5.27 \\
\hline 20 & 24 & 3 & 628 & R caudate & -3.70 \\
\hline 36 & 12 & -27 & 278 & R temporal pole & -3.51 \\
\hline-60 & -33 & 41 & 295 & L supramarginal gyrus & -3.37 \\
\hline-2 & 42 & 29 & 426 & $\begin{array}{l}\mathrm{L} \text { anterior cingulate } \\
\text { cortex }\end{array}$ & -3.28 \\
\hline
\end{tabular}

Abbreviations: $L$ left, $M N I$ the Montreal Neurological Institute, $R$ right, $T H$ tension-type headache
In the post-hoc analysis (Table 3 and Fig. 3), the episodic TTH vs. episodic migraine groups demonstrated a higher GM volume in the bilateral putamen, right caudate, middle frontal gyrus, and cerebellum. Conversely, GM volume of the right lateral occipital cortex was lower in episodic TTH compared with episodic migraine. A comparison of the GM volume between chronic TTH and chronic migraine only revealed a higher GM volume of the left cerebellum in chronic TTH.

\section{Predictive value of GM volume difference for headache types}

A logistic regression model was employed to assess whether the GM volume differences between TTH and migraine could predict headache types (TTH vs. migraine). After adjustment for age, sex, and depression, two of the brain regions indicating a headache-type effect $(\mathrm{TTH}>\mathrm{mi}-$ graine; Table 3 and Fig. 2) were predictive of TTH diagnosis: the left superior frontal gyrus (beta $=15.92, P=0.001$ ) and right cerebellum $\mathrm{V}$ (beta $=10.33, P=0.006$ ). In the ROC analysis, to distinguish TTH from migraine, the area under the curve of the regression model was 0.806 , indicating good discrimination (Fig. 4).

\section{Discussion}

Structural MRI and VBM were used to compare GM volume between migraine and "strict-criteria" TTH. We determined that TTH and migraine differed in brain morphology because (1) the GM volume of specific brain regions were increased (anterior cingulate cortex, supramarginal gyrus, temporal pole, lateral occipital cortex, and caudate) in patients with TTH whereas decreased (orbitofrontal cortex) in patients with migraine compared with healthy controls; (2) a direct comparison of GM volume between both headache disorders revealed a lower GM volume in the superior and middle frontal gyri, cerebellum, dorsal striatum (putamen and caudate), and precuneus in patients with migraine; (3) the GM of the left superior frontal gyrus and right cerebellum $\mathrm{V}$ together demonstrated good discriminative ability for $\mathrm{TTH}$ and migraine in the ROC analysis. In addition, headache chronification in patients with TTH and migraine was associated with a GM decrease in the bilateral insula and anterior cingulate cortex. Although the neurobiological basis for these GM plastic changes is not sufficiently understood, we discuss herein their potential relevance for TTH and migraine pathophysiology.

\section{GM increase in TTH and decrease in migraine (vs. controls)}

Earlier MRI studies on TTH have revealed no volumetric GM change in episodic TTH [20] and reduced GM in chronic TTH in the anterior cingulate, insula, 

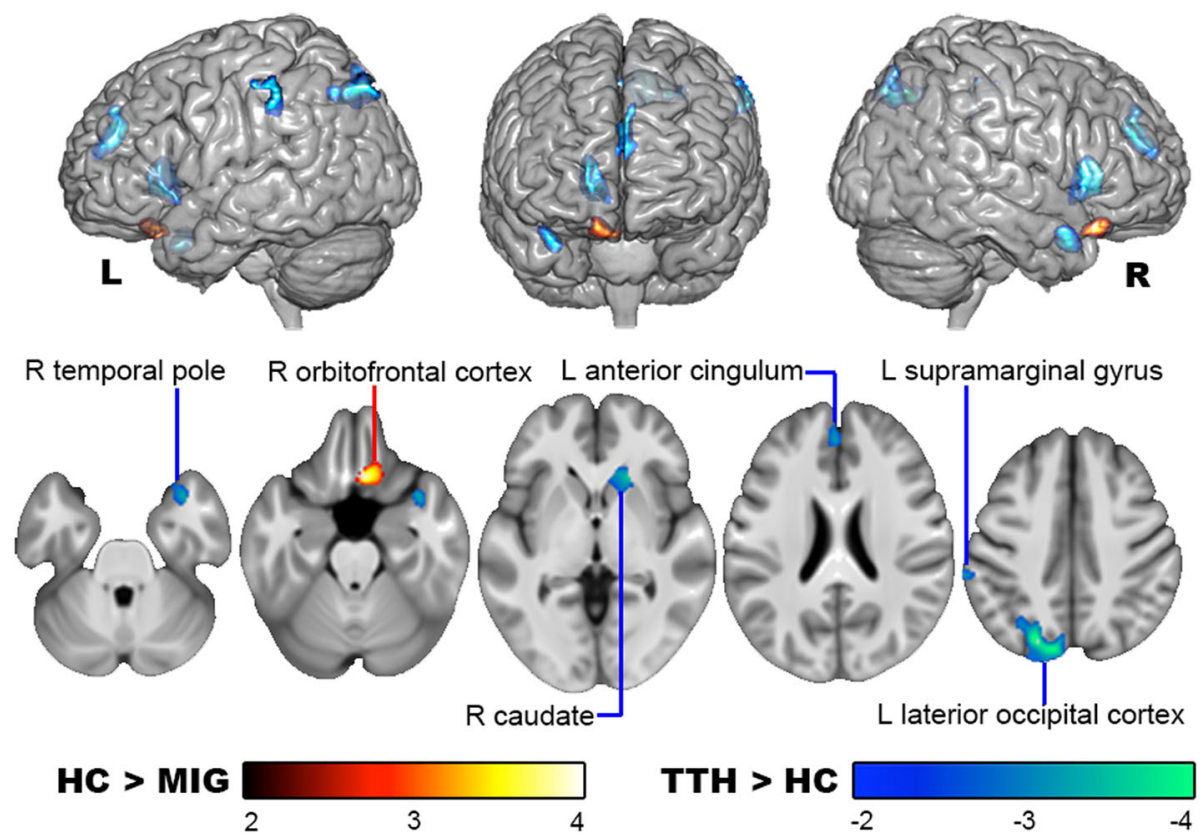

TTH > HC

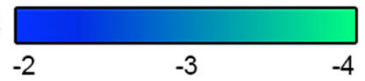

Fig. 1 Altered gray matter volume in TTH and migraine. In comparison with controls, the patients with TTH exhibited a gray matter volume increase in the anterior cingulate cortex, supramarginal gyrus, temporal pole, lateral occipital cortex, and caudate. The patients with migraine conversely exhibited a gray matter volume decrease in the orbitofrontal cortex. HC: healthy controls; L: left; MIG: migraine; R: right; TTH: tension-type headache

orbitofrontal cortex, dorsal pons, and other brain structures involved in pain processing [9]. Our findings of increased GM volume in TTH (also in episodic and chronic subgroups) appear to conflict with earlier studies, although the brain regions that exhibited a GM increase are also part of the pain processing network [27]. In addition to the adjustment for the depression effect and different $P$ value thresholds, the strict TTH criteria employed in this study may be the main reason for this discrepancy. For patients with migraine, this study was in line with the literature $[10,12]$ indicating reduced GM in the orbitofrontal cortex, a region associated with reward, adaptive behavior, and pain processing [28]. Notably, most migraine studies on GM change, despite heterogeneous findings in localization, have congruently demonstrated reduced GM in the pain processing network $[10,11,13,29-31]$.

\section{GM difference between TTH and migraine}

One notable finding of this study was that TTH and migraine differed in the GM volumes of several prefrontal and cerebellar regions (Fig. 2), and both types of headaches could be distinguished by the volumes of the left superior frontal gyrus and right cerebellum $\mathrm{V}$ in ROC analysis. In meta-analyses, the prefrontal cortex was identified as being the most crucial brain area associated with structural change in migraine [32, 33]. A discriminative analysis of migraine without aura (vs. controls) also identified, using a machine learning classifier, the superior frontal gyrus as one of the most discriminative GM features [34]. The present finding of a lower prefrontal GM volume in patients with migraine has three clinical implications. First, the prefrontal cortex is associated with the descending inhibitory mechanism of pain modulation [35]. A GM decrease in this region may be linked to inhibitory dysfunction and hence a heightened severity of migraine versus TTH. Second, the superior and middle frontal gyri are involved in task monitoring and temporal organization, two crucial aspects of executive function [36]. GM decrease in these prefrontal regions was linked to executive dysfunction (i.e., delayed response time to task set-shifting) in patients with migraine [37]. Thus, lower prefrontal GM in patients with migraine may reflect a greater extent of cognitive dysfunction in migraine versus TTH [38]. Third, a higher prevalence of psychiatric comorbidities in patients with migraine versus those with TTH [39] may also partly be explained by the lower prefrontal GM in patients with migraine. The discriminative ability of cerebellum for TTH and migraine appears to correspond with most studies that indicated a GM decrease $[31,33,40]$ and subclinical dysfunction [41-44] of the cerebellum in patients with migraine. The cerebellum also plays an inhibitory role in nociception, given its extensive connection with the prefrontal cortex [45]. Thus, the relative GM decrease in the cerebellum may be partly related to heighted pain severity in migraine. 
Table 3 A $2 \times 2$ ANCOVA analysis for the gray matter volume difference between $\mathrm{TH}$ and migraine

\begin{tabular}{|c|c|c|c|c|c|}
\hline \multicolumn{3}{|c|}{ MNI coordinates } & \multirow{2}{*}{$\begin{array}{l}\text { Cluster } \\
\text { size }\end{array}$} & \multirow[t]{2}{*}{ Anatomical region } & \multirow[t]{2}{*}{ Local peak T/F-value } \\
\hline$x$ & $y$ & z & & & \\
\hline \multicolumn{6}{|c|}{ Headache type effect (TTH > migraine) } \\
\hline-27 & -38 & -39 & 705 & L cerebellum VI & 4.57 \\
\hline 5 & -74 & 50 & 380 & R precuneus cortex & 4.38 \\
\hline-24 & 8 & 65 & 611 & $\begin{array}{l}\text { L superior frontal } \\
\text { gyrus }\end{array}$ & 4.23 \\
\hline 3 & -87 & -26 & 590 & R cerebellum crus $\|$ & 4.03 \\
\hline 32 & -17 & 6 & 1839 & R putamen & 3.81 \\
\hline 32 & -33 & -33 & 340 & R cerebellum $V$ & 3.75 \\
\hline 34 & 18 & 59 & 324 & $\mathrm{R}$ middle frontal gyrus & 3.55 \\
\hline-29 & -20 & 3 & 1128 & L putamen & 3.55 \\
\hline 17 & 13 & 8 & 638 & R caudate & 3.45 \\
\hline
\end{tabular}

Headache frequency effect (Episodic > Chronic)

$\begin{array}{llllll}14 & 45 & 15 & 933 & \begin{array}{l}\text { R anterior cingulate } \\ \text { cortex }\end{array} & 3.96 \\ -47 & 17 & 0 & 1070 & L \text { insula } & 3.87 \\ -6 & -57 & -2 & 366 & L \text { cerebellum V } & 3.78 \\ 36 & 29 & 7 & 364 & \text { R insula } & 3.54\end{array}$

Type $\times$ frequency interaction effect

$\begin{array}{lllll}29 & -72 & 24 & 530 \quad \text { R lateral occipital } & 14.04\end{array}$ cortex

Post-hoc comparisons

Episodic TTH > Episodic migraine

$\begin{array}{llllll}26 & 14 & 1 & 1850 & \text { R putamen } & 4.21 \\ 3 & -86 & -21 & 542 & \text { R cerebellum crus I } & 3.91 \\ 41 & 26 & 51 & 573 & \begin{array}{l}\text { R middle frontal } \\ \text { Gyrus }\end{array} & 3.91 \\ -29 & -18 & 0 & 1131 & \text { L putamen } & 3.87 \\ 9 & 12 & 14 & 364 & \text { R caudate } & 3.30\end{array}$

Episodic TTH < episodic migraine

$\begin{array}{llllll}42 & -78 & -12 & 535 & \text { R lateral occipital } & -3.55\end{array}$

$$
\text { cortex }
$$

Chronic TTH > Chronic migraine

$\begin{array}{lllll}-27 & -41 & -38 & 640 \quad \mathrm{~L} \text { cerebellum } \mathrm{Vl}\end{array}$

Chronic TTH < Chronic migraine: non-significant

Abbreviations: $L$ left, $M N I$ Montreal Neurological Institute, N.S. non-significant, $R$ right, $\Pi \mathrm{TH}$ tension-type headache

The dorsal striatum and precuneus also differed between patients with TTH and migraine in GM volume. The dorsal striatum is part of the pain processing network, and its activation may encode pain intensity [46]. The difference in striatal volume between TTH and migraine may be explained by a GM increase in patients with TTH (vs. controls, Fig. 1) and a tendency for migraine to reduce GM in this region [29, 47]. The precuneus is a pivotal region of the default mode network, which was particularly sensitive to the cognitive states in self-referential tasks [48]. The functional connectivity of the default mode network was changed in various pain conditions [49], and the connectivity of the network with insula has been reported to encode pain intensity $[50,51]$. The relative GM decrease in $\mathrm{mi}$ graine (vs. TTH) is thus explicable by the extent to which migraine interferes with information processing and intrinsic variation in brain activity. We are not sure why the lateral occipital cortex is increased in GM in episodic migraine (vs. episodic TTH) and in TTH (vs. controls). However, this brain region, in addition to its well-known involvement in visual processing, is associated with cognitive evaluation of pain [52], and has been linked to abnormal emotional processing and self-focused attention [53]. An earlier MRI study on episodic migraine also showed increased cortical thickness and gyrification index in this region [54].

\section{GM change associated with headache frequency}

That the GM of the anterior cingulate cortex and insula decreases as a headache evolves from the episodic to chronic form is not unexpected (headache frequency effect). The anterior cingulate cortex and insula are the pivotal relay regions of the pain network and involved in the affective and cognitive processing of subjective pain experience $[27,46]$. Notably, their GM decreases are not specific to chronic headaches but have also been reported in various types of chronic pain including fibromyalgia [55], classical trigeminal neuralgia [56], and phantom limb pain [57]. These plastic changes may underpin the common emotional distress and cognitive dysfunction that cause disability in patients with chronic pain [58]. Moreover, the findings of this study suggest that the structural difference between TTH and migraine, as judged by the number of brain regions (Fig. 3), is more prominent in their episodic forms than chronic forms. The trend toward a structural similarity in the chronification of TTH and migraine may resemble the clinical scenario, in which chronic migraine often loses its vegetative characteristics (accompanying photophobia, phonophobia, nausea, vomiting, and headache exacerbation with physical exercise) and thus resembles TTH [59].

\section{GM change and allostatic load}

The mechanism of GM volume change is beyond the scope of this study. However, the present findings of GM change in TTH and migraine (vs. controls) and the GM difference between both types of headaches mostly involved brain regions of the pain processing network, which suggested these plastic changes may reflect the allostatic load in response to headache pain [60]. It is posited that the pain processing network may present an adaptive volume increase to mild- 

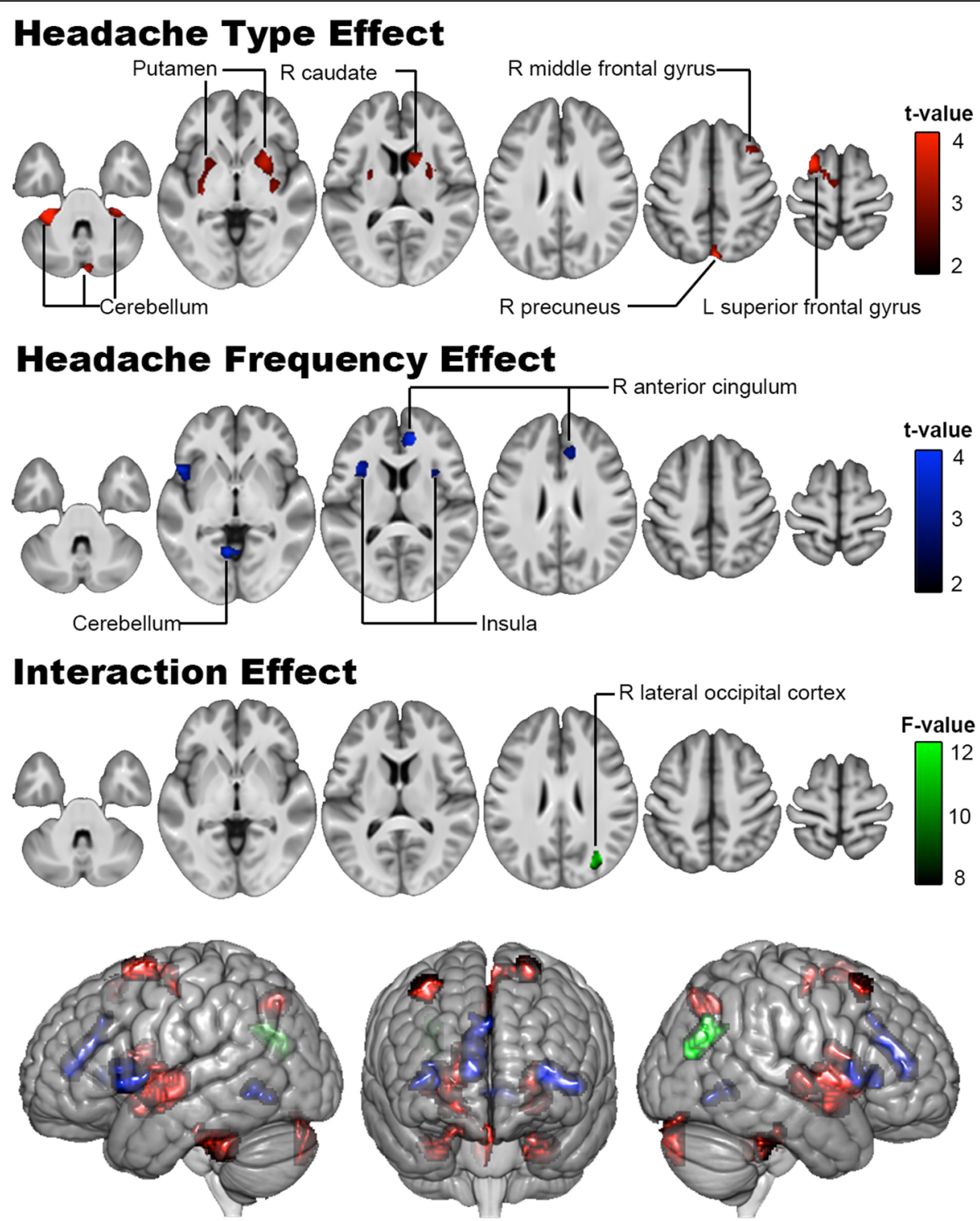

Fig. 2 Difference of gray matter volume between TTH and migraine. A $2 \times 2$ ANCOVA analysis was used to investigate the effects of headache types (TTH vs. migraine), headache frequency (episodic vs. chronic) and their interaction upon the gray matter volume difference between TTH and migraine. The brain regions with gray matter differences are color-coded in red (TTH > migraine), blue (episodic $>$ chronic) and green (type $\times$ frequency interaction). L: left; MIG: migraine; R: right; TTH: tension-type headache

to-moderate TTH pain, whereas a maladaptive volume decrease to the moderate-to-severe migraine pain. Notably, a pain severity-dependent plastic change in GM volume has been reported in chronic migraine and phantom limb pain $[61,62]$. Earlier evoked potential studies that showed a deficient habituation in migraine in contrast with a relatively intact habituation in TTH also suggest a greater allostatic load in migraine (vs. TTH), hence a maladaptive brain response [63].

\section{Study limitations}

This study was limited in terms of the generalizability of its findings to patients with migraine with aura or medication overuse and patients with TTH diagnosed according to the standard ICHD-III beta criteria. Moreover, our findings cannot be generalized to the ictal imaging data because dynamic GM change across the ictal-interictal cycle has been reported in TTH [20] and migraine [64]. The technique of VBM has inherent limitations [65]. Histological measures such as neuronal density do not correlate with VBM GM probability maps [66]. Changes in cerebral blood flow may apparently change GM volume in VBM analyses [67]. Our findings did not include areas that were specifically activated in migraine (i.e., the brainstem and hypothalamus [68]). However, the negative finding does not imply that the 

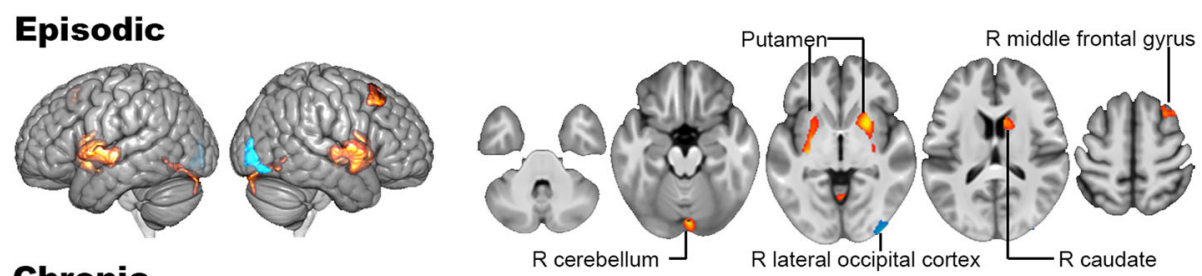

Chronic

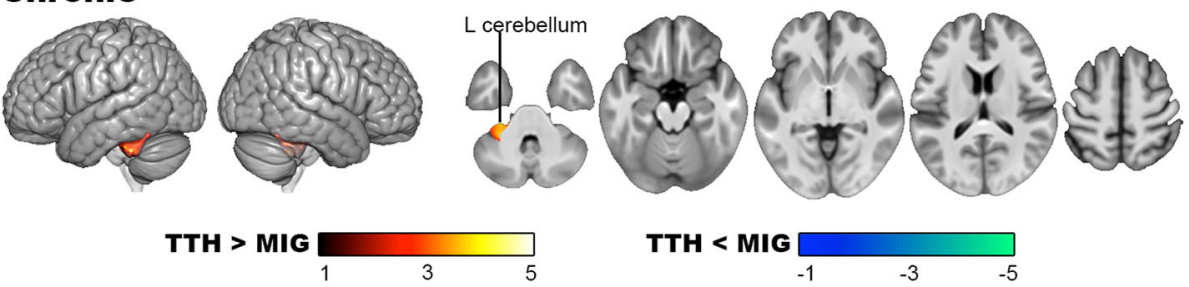

Fig. 3 Post-hoc analysis for the gray matter volume difference between TTH and migraine $(2 \times 2$ ANCOVA). The episodic TTH vs. episodic migraine groups demonstrated a higher gray matter volume in the bilateral putamen, right caudate, middle frontal gyrus, and cerebellum. Conversely, gray matter volume of the right lateral occipital cortex was lower in episodic TTH compared with episodic migraine. A comparison of gray matter volume between chronic TTH and chronic migraine only revealed a higher gray matter volume of the left cerebellum in chronic TTH. L: left; MIG: migraine; R: right; TTH: tension-type headache

brainstem and hypothalamus have no role in differentiating between TTH and migraine in brain morphology, because these brain regions are relatively small for a whole-brain analysis. The complex tissue pattern in these deep structures may also hamper precision of the VBM-based tissue segmentation. Further 3 T MRI studies using methods refined for analyzing these specific structures are warranted to draw conclusions. Limited by its design, the present

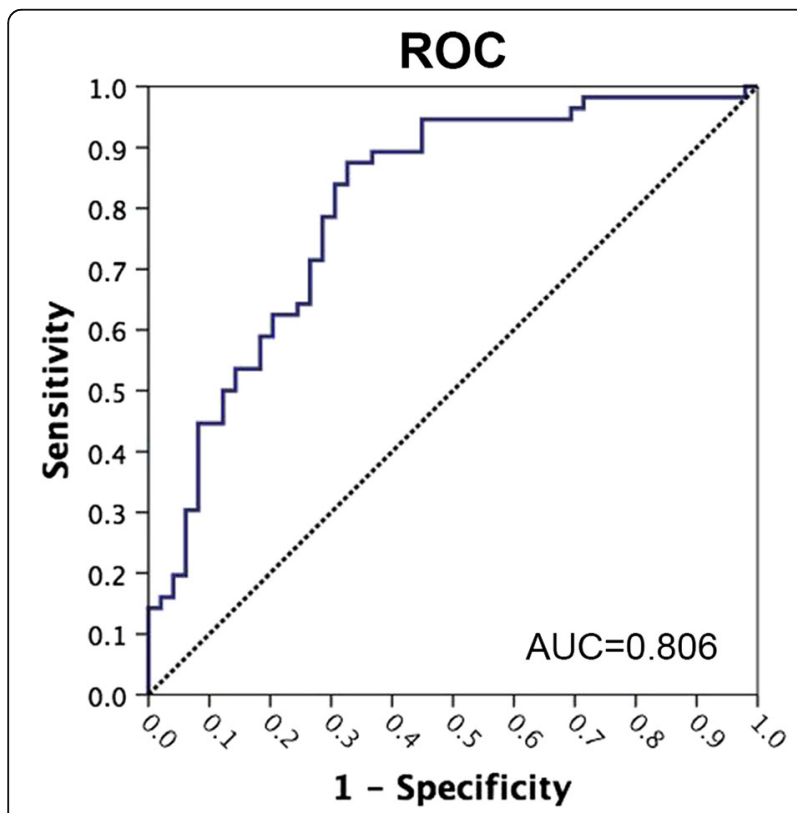

Fig. 4 Receiver operating characteristic (ROC) analysis to distinguish TTH from migraine. In ROC analysis, the gray matter volumes of the left superior frontal gyrus and right cerebellum $V$ combined had excellent discriminative ability for distinguishing $\mathrm{TTH}$ and migraine (area under the curve $[\mathrm{AUC}]=0.806$ ) study could not elucidate the causal relationship between brain morphological change and headache phenotypes. A longitudinal study in the same patient group would be particularly valuable to examine whether the GM difference between TTH and migraine is a steady phenotypic marker independent of disease duration. Finally, the diagnostic ability of the GM volume to differentiate between TTH and migraine must be confirmed in a new patient population.

\section{Conclusions}

TTH and migraine are separate headache disorders with different characteristics of GM change. The major morphological difference between the two types of headaches is a relative GM decrease in the prefrontal and cerebellar regions in migraine, which may reflect a higher allostatic load associated with this disabling headache. Our findings may facilitate the development of a TTH-specific treatment and phenotypic marker. However, these GM changes remain undetermined in the neurobiological mechanism, temporal stability, and causal relationship with headache phenotypes.

\section{Additional files}

Additional file 1: Table S1. Demographics and clinical profile of the five participant groups (DOCX $17 \mathrm{~kb}$ )

Additional file 2: Figure S1. Altered gray matter volume in episodic and chronic TTH. In the subgroup analysis, gray matter volume (vs. controls) was unaltered in the episodic and chronic migraine groups, but was increased in specific brain regions in patients with episodic and chronic TTH. CTTH: chronic tension-type headache; ETTH: episodic tension-type headache; HC: healthy controls; L: left; R: right. (TIFF 2705 kb)

\section{Abbreviations}

ANCOVA: Analysis of covariance; BDI: The Beck Depression Inventory; CGRP: Calcitonin gene-related peptide; GM: Gray matter; ICHD: International Classification of Headache Disorders; MIDAS: Migraine Disability Assessment; 
MNI: The Montreal Neurological Institute; MRI: Magnetic resonance imaging; ROC: Receiver operating characteristic; ROI: Region of interest; $T \mathrm{TH}$ : Tensiontype headache; VBM: Voxel-based morphometry

\section{Acknowledgements}

We would like to thank the study participants and the research assistants for actively participating.

\section{Funding}

This study was supported by grants from Ministry of Science and Technology (NSC101-2314-B-075-068-MY2, MOST 106-2321-B-010-009-, and MOST 1062314-B-075-026- to WT Chen; 103-2321-B-010-017, 102-2321-B-010-030, 1002314-B-010-018-MY3, 104-2745-B-010-003 and MOST 106-2321-B-010-009 to SJ Wang), Taipei Veterans General Hospital (V105C-092, V105E9-005-MY2-1, and VGHUST104-G7-1-3 to WT Chen; VGHUST105-G7-1-1, V105C-127, V105E9-001MY2-1, and VTA105-V1-1-1 to SJ Wang), Brain Research Center, National YangMing University, a grant from Ministry of Education, Aim for the Top University Plan, and grants from the Ministry of Health and Welfare (MOHW 106-TDU-B211-113001).

\section{Availability of data and materials}

We have uploaded all the un-thresholded statistical maps of the present imaging data to the NeuroVault website, available through the following permanent link: https://neurovault.org/collections/3198/.

\section{Authors' contributions}

WT Chen was the project leader and contributed to project design and development of the methodology. KL Lai, JL Fuh, and SJ Wang enrolled and evaluated the patients. KH Chou, PL Lee, FJ Hsiao, DM Niddam, and CP Lin collected and analyzed the imaging data. WT Chen and KH Chou drafted the manuscript. SJ Wang revised the manuscript critically. All authors read and approved the final manuscript.

\section{Authors' information}

None

\section{Ethics approval and consent to participate}

The Institutional Review Board of Taipei Veterans General Hospital approved the study protocol (Reference No. 2012-07-013 AC) and each participant provided written informed consent.

\section{Consent for publication}

Not applicable.

\section{Competing interests}

WT Chen, KH Chou, PL Lee, FJ Hsiao, DM Niddam, KL Lai, and CP Lin report no competing interests. SJ Wang has served on the advisory boards of Allergan, and Eli Lilly Taiwan. He has received honoraria as a moderator from local companies (Taiwan branches) of Pfizer, Eli Lilly and Esai. JL Fuh is a member of the scientific advisory board of Novartis, and has received research support from the Taiwan National Science Council and Taipei-Veterans General Hospital.

\section{Publisher's Note}

Springer Nature remains neutral with regard to jurisdictional claims in published maps and institutional affiliations.

\section{Author details}

'Department of Neurology, School of Medicine, National Yang-Ming University, Taipei, Taiwan. ${ }^{2}$ The Neurological Institute, Taipei Veterans General Hospital, Taipei, Taiwan. ${ }^{3}$ Brain Research Center, National Yang-Ming University, Taipei, Taiwan. ${ }^{4}$ Institute of Brain Science, School of Medicine, National Yang-Ming University, Taipei, Taiwan. ${ }^{5}$ Institute of Neuroscience, National Yang-Ming University, Taipei, Taiwan. ${ }^{6}$ Department of Biomedical Imaging and Radiological Sciences, National Yang-Ming University, Taipei, Taiwan.
Received: 4 December 2017 Accepted: 2 January 2018

Published online: 15 January 2018

\section{References}

1. Lyngberg AC, Rasmussen BK, Jorgensen T, Jensen R (2005) Has the prevalence of migraine and tension-type headache changed over a 12-year period? A Danish population survey. Eur J Epidemiol 20:243-249

2. Disease GBD, Injury I, Prevalence C (2017) Global, regional, and national incidence, prevalence, and years lived with disability for 328 diseases and injuries for 195 countries, 1990-2016: a systematic analysis for the global burden of disease study 2016. Lancet 390:1211-1259

3. Headache Classification Committee of the International Headache Society (2013) The international classification of headache disorders, 3rd edition (beta version). Cephalalgia 33:629-808

4. Vargas BB (2008) Tension-type headache and migraine: two points on a continuum? Curr Pain Headache Rep 12:433-436

5. Lipton RB, Cady RK, Stewart WF, Wilks K, Hall C (2002) Diagnostic lessons from the spectrum study. Neurology 58:S27-S31

6. Turner DP, Smitherman TA, Black AK, Penzien DB, Porter JAH, Lofland KR, Houle TT (2015) Are migraine and tension-type headache diagnostic types or points on a severity continuum? An exploration of the latent taxometric structure of headache. Pain 156:1200-1207

7. Lai TH, Protsenko E, Cheng YC, Loggia ML, Coppola G, Chen WT (2015) Neural plasticity in common forms of chronic headaches. Neural Plast 2015: 205985

8. de Tommaso M, Ambrosini A, Brighina F, Coppola G, Perrotta A, Pierelli F, Sandrini G, Valeriani M, Marinazzo D, Stramaglia S, Schoenen J (2014) Altered processing of sensory stimuli in patients with migraine. Nat Rev Neurol 10:144-155

9. Schmidt-Wilcke T, Leinisch E, Straube A, Kampfe N, Draganski B, Diener HC, Bogdahn U, May A (2005) Gray matter decrease in patients with chronic tension type headache. Neurology 65:1483-1486

10. Kim JH, Suh SI, Seol HY, Oh K, Seo WK, Yu SW, Park KW, Koh SB (2008) Regional grey matter changes in patients with migraine: a voxel-based morphometry study. Cephalalgia 28:598-604

11. Rocca MA, Ceccarelli A, Falini A, Colombo B, Tortorella P, Bernasconi L, Comi G, Scotti G, Filippi M (2006) Brain gray matter changes in migraine patients with T2-visible lesions: a 3-T MRI study. Stroke 37:1765-1770

12. Rocca MA, Messina R, Colombo B, Falini A, Comi G, Filippi M (2014) Structural brain MRI abnormalities in pediatric patients with migraine. J Neurol 261:350-357

13. Schmidt-Wilcke T, Ganssbauer S, Neuner T, Bogdahn U, May A (2008) Subtle grey matter changes between migraine patients and healthy controls. Cephalalgia 28:1-4

14. Ashina M, Bendtsen L, Jensen R, Schifter S, Jansen-Olesen I, Olesen J (2000) Plasma levels of calcitonin gene-related peptide in chronic tension-type headache. Neurology 55:1335-1340

15. Andersen S, Petersen MW, Svendsen AS, Gazerani P (2015) Pressure pain thresholds assessed over temporalis, masseter, and frontalis muscles in healthy individuals, patients with tension-type headache, and those with migraine-a systematic review. Pain 156:1409-1423

16. Avramidis T, Bougea A, Hadjigeorgiou G, Thomaides T, Papadimitriou A (2017) Blink reflex habituation in migraine and chronic tension-type headache. Neurol Sci. 38:993-998

17. Valeriani M, de Tommaso M, Restuccia D, Le Pera D, Guido M, lannetti GD, Libro G, Truini A, Di Trapani G, Puca F, Tonali P, Cruccu G (2003) Reduced habituation to experimental pain in migraine patients: a $\mathrm{CO}(2)$ laser evoked potential study. Pain 105:57-64

18. Vuralli D, Boran HE, Cengiz B, Coskun O, Bolay H (2017) Somatosensory temporal discrimination remains intact in tension-type headache whereas it is disrupted in migraine attacks. Cephalalgia 37:1241-1247

19. Smallwood RF, Laird AR, Ramage AE, Parkinson AL, Lewis J, Clauw DJ, Williams DA, Schmidt-Wilcke T, Farrell MJ, Eickhoff SB, Robin DA (2013) Structural brain anomalies and chronic pain: a quantitative meta-analysis of gray matter volume. J Pain 14:663-675

20. Chen B, He Y, Xia L, Guo LL, Zheng JL (2016) Cortical plasticity between the pain and pain-free phases in patients with episodic tension-type headache. $J$ Headache Pain 17:105

21. Hung PH, Fuh JL, Wang SJ (2006) Validity, reliability and application of the taiwan version of the migraine disability assessment questionnaire. J Formos Med Assoc 105:563-568 
22. Beck AT, Steer RA, Ball R, Ranieri W (1996) Comparison of Beck depression inventories-IA and -II in psychiatric outpatients. J Pers Assess 67:588-597

23. Ashburner J, Friston KJ (2000) Voxel-based morphometry-the methods. Neurolmage 11:805-821

24. Lai TH, Chou KH, Fuh JL, Lee PL, Kung YC, Lin CP, Wang SJ (2016) Gray matter changes related to medication overuse in patients with chronic migraine. Cephalalgia 36:1324-1333

25. Yang FC, Chou KH, Fuh JL, Huang CC, Lirng JF, Lin YY, Lin CP, Wang SJ (2013) Altered gray matter volume in the frontal pain modulation network in patients with cluster headache. Pain 154:801-807

26. Ashburner J (2007) A fast diffeomorphic image registration algorithm. Neurolmage 38:95-113

27. Peyron R, Laurent B, Garcia-Larrea L (2000) Functional imaging of brain responses to pain. A review and meta-analysis (2000). Neurophysiol Clin 30: 263-288

28. Rolls ET (2000) The orbitofrontal cortex and reward. Cereb Cortex 10: 284-294

29. Schmitz N, Admiraal-Behloul F, Arkink EB, Kruit MC, Schoonman GG, Ferrari MD, van Buchem MA (2008) Attack frequency and disease duration as indicators for brain damage in migraine. Headache 48:1044-1055

30. Valfre W, Rainero I, Bergui M, Pinessi L (2008) Voxel-based morphometry reveals gray matter abnormalities in migraine. Headache 48:109-117

31. Jin C, Yuan K, Zhao L, Zhao L, Yu D, von Deneen KM, Zhang M, Qin W, Sun W, Tian J (2013) Structural and functional abnormalities in migraine patients without aura. NMR Biomed 26:58-64

32. Hu W, Guo J, Chen N, Guo J, He L (2015) A meta-analysis of voxel-based morphometric studies on migraine. Int J Clin Exp Med 8:4311-4319

33. Jia Z, Yu S (2017) Grey matter alterations in migraine: a systematic review and meta-analysis. Neuroimage Clin 14:130-140

34. Zhang Q, Wu Q, Zhang J, He L, Huang J, Zhang J, Huang H, Gong Q (2016) Discriminative analysis of migraine without Aura: using functional and structural MRI with a multi-feature classification approach. PLoS One 11: e0163875

35. Lorenz J, Minoshima S, Casey KL (2003) Keeping pain out of mind: the role of the dorsolateral prefrontal cortex in pain modulation. Brain 126:10791091

36. Fassbender C, Murphy K, Foxe JJ, Wylie GR, Javitt DC, Robertson $H_{\text {, Garavan }}$ $H$ (2004) A topography of executive functions and their interactions revealed by functional magnetic resonance imaging. Brain research. Cogn Brain Res 20:132-143

37. Schmitz N, Arkink EB, Mulder M, Rubia K, Admiraal-Behloul F, Schoonman GG, Kruit MC, Ferrari MD, van Buchem MA (2008) Frontal lobe structure and executive function in migraine patients. Neurosci Lett 440:92-96

38. O'Bryant SE, Marcus DA, Rains JC, Penzien DB (2006) The neuropsychology of recurrent headache. Headache 46:1364-1376

39. Mongini F, Rota E, Deregibus A, Ferrero L, Migliaretti G, Cavallo F, Mongini T, Novello A (2006) Accompanying symptoms and psychiatric comorbidity in migraine and tension-type headache patients. J Psychosom Res 61:447-451

40. Bilgic B, Kocaman G, Arslan AB, Noyan H, Sherifov R, Alkan A, Asil T, Parman $Y$, Baykan B (2016) Volumetric differences suggest involvement of cerebellum and brainstem in chronic migraine. Cephalalgia 36:301-308

41. Gerwig M, Rauschen L, Gaul C, Katsarava Z, Timmann D (2014) Subclinical cerebellar dysfunction in patients with migraine: evidence from eyeblink conditioning. Cephalalgia 34:904-913

42. Ishizaki K, Mori N, Takeshima T, Fukuhara Y, ljiri T, Kusumi M, Yasui K, Kowa H, Nakashima K (2002) Static stabilometry in patients with migraine and tension-type headache during a headache-free period. Psychiatry Clin Neurosci 56:85-90

43. Sandor PS, Mascia A, Seidel L, de Pasqua V, Schoenen J (2001) Subclinical cerebellar impairment in the common types of migraine: a threedimensional analysis of reaching movements. Ann Neurol 49:668-672

44. Harno H, Hirvonen $T$, Kaunisto MA, Aalto H, Levo H, Isotalo E, Kallela M, Kaprio J, Palotie A, Wessman M, Farkkila M (2003) Subclinical vestibulocerebellar dysfunction in migraine with and without aura. Neurology 61:1748-1752

45. Ruscheweyh R, Kuhnel M, Filippopulos F, Blum B, Eggert T, Straube A (2014) Altered experimental pain perception after cerebellar infarction. Pain 155: 1303-1312

46. Wilcox CE, Mayer AR, Teshiba TM, Ling J, Smith BW, Wilcox GL, Mullins PG (2015) The subjective experience of pain: an FMRI study of percept-related models and functional connectivity. Pain Med 16:2121-2133
47. Maleki N, Becerra L, Nutile L, Pendse G, Brawn J, Bigal M, Burstein R, Borsook D (2011) Migraine attacks the basal ganglia. Mol Pain 7:71

48. Raichle ME, MacLeod AM, Snyder AZ, Powers WJ, Gusnard DA, Shulman GL (2001) A default mode of brain function. Proc Natl Acad Sci U S A 98:676-682

49. Baliki MN, Mansour AR, Baria AT, Apkarian AV (2014) Functional reorganization of the default mode network across chronic pain conditions. PLoS One 9: e106133

50. Loggia ML, Kim J, Gollub RL, Vangel MG, Kirsch I, Kong J, Wasan AD, Napadow V (2013) Default mode network connectivity encodes clinical pain: an arterial spin labeling study. Pain 154:24-33

51. Napadow V, LaCount L, Park K, As-Sanie S, Clauw DJ, Harris RE (2010) Intrinsic brain connectivity in fibromyalgia is associated with chronic pain intensity. Arthritis Rheum 62:2545-2555

52. Kong J, White NS, Kwong KK, Vangel MG, Rosman IS, Gracely RH, Gollub RL (2006) Using fMRI to dissociate sensory encoding from cognitive evaluation of heat pain intensity. Hum Brain Mapp 27:715-721

53. Frick A, Engman J, Alaie I, Bjorkstrand J, Faria V, Gingnell M, Wallenquist U, Agren T, Wahlstedt K, Larsson EM, Morell A, Fredrikson M, Furmark T (2014) Enlargement of visual processing regions in social anxiety disorder is related to symptom severity. Neurosci Lett 583:114-119

54. Zhang J, YL W, Su J, Yao Q, Wang M, Li GF, Zhao R, Shi YH, Zhao Y, Zhang Q, Lu H, Xu S, Qin Z, Cui GH, Li J, Liu JR, Du X (2017) Assessment of gray and white matter structural alterations in migraineurs without aura. J Headache Pain 18:74

55. Robinson ME, Craggs JG, Price DD, Perlstein WM, Staud R (2011) Gray matter volumes of pain-related brain areas are decreased in fibromyalgia syndrome. J Pain 12:436-443

56. Wang Y, Cao DY, Remeniuk B, Krimmel S, Seminowicz DA, Zhang M (2017) Altered brain structure and function associated with sensory and affective components of classic trigeminal neuralgia. Pain 158:1561-1570

57. Draganski B, Moser T, Lummel N, Ganssbauer S, Bogdahn U, Haas F, May A (2006) Decrease of thalamic gray matter following limb amputation. Neurolmage 31:951-957

58. Malfliet A, Coppieters I, Van Wilgen P, Kregel J, De Pauw R, Dolphens M, Ickmans K (2017) Brain changes associated with cognitive and emotional factors in chronic pain: a systematic review. Eur J Pain 21:769-786

59. May A, Schulte LH (2016) Chronic migraine: risk factors, mechanisms and treatment. Nat Rev Neurol 12:455-464

60. Borsook D, Maleki N, Becerra L, McEwen B (2012) Understanding migraine through the lens of maladaptive stress responses: a model disease of allostatic load. Neuron 73:219-234

61. Preissler S, Feiler J, Dietrich C, Hofmann GO, Miltner WH, Weiss T (2013) Gray matter changes following limb amputation with high and low intensities of phantom limb pain. Cereb Cortex 23:1038-1048

62. Chen Z, Chen X, Liu M, Liu S, Ma L, Yu S (2017) Volume expansion of periaqueductal gray in episodic migraine: a pilot MRI structural imaging study. J Headache Pain 18:83

63. Coppola G, Di Lorenzo C, Schoenen J, Pierelli F (2013) Habituation and sensitization in primary headaches. J Headache Pain 14:65

64. Coppola G, Di Renzo A, Tinelli E, lacovelli E, Lepre C, Di Lorenzo C, Di Lorenzo G, Di Lenola D, Parisi V, Serrao M, Pauri F, Fiermonte G, Bianco F, Pierelli $F$ (2015) Evidence for brain morphometric changes during the migraine cycle: a magnetic resonance-based morphometry study. Cephalalgia 35:783-791

65. May A (2009) Morphing voxels: the hype around structural imaging of headache patients. Brain 132:1419-1425

66. Eriksson SH, Free SL, Thom M, Symms MR, Martinian L, Duncan JS, Sisodiya SM (2009) Quantitative grey matter histological measures do not correlate with grey matter probability values from in vivo MRI in the temporal lobe. J Neurosci Methods 181:111-118

67. Franklin TR, Wang Z, Shin J, Jagannathan K, Suh JJ, Detre JA, O'Brien CP, Childress AR (2013) A VBM study demonstrating 'apparent' effects of a single dose of medication on T1-weighted MRIs. Brain Struct Funct 218:97-104

68. Denuelle M, Fabre N, Payoux P, Chollet F, Geraud G (2007) Hypothalamic activation in spontaneous migraine attacks. Headache 47:1418-1426 